\title{
Adaptive Compression Scheme for Video Delivery over the QoS-aware Residential Gateway
}

\author{
Pei-Chen Tseng, *Ching-Tuey Sung, and Wen-Shyang Hwang \\ National Kaohsiung University of Applied Sciences \\ *Tainan University of Technology \\ E-mail:peichen@wshlab2.ee.kuas.edu.tw,t00046@ms.twcat.edu.tw, and \\ wshwang@mail.ee.kuas.edu.tw
}

\begin{abstract}
A simple adaptive subsampling/de-subsampling scheme for video delivery over the QoS-aware residential gateway $(Q R G)$ during network congestion is presented. The network performs better by transmitting fewer data by fewer bits required during network congestion. The proposed subsampling scheme can be adaptively adjust with variable compression ratio using different kind of subsampling algorithms according to the feedback information from the $Q R G$; no subsampling is allowed in normal operation, which provided sufficient network bandwidth; intra-frame subsampling scheme is adopted for medium, and interframe subsampling scheme for heavily traffic during network congestion. The receiver adaptively desubsamples the subsampled frame with a simple linear interpolation to reconstruct the image-frame. QRG is experimentally implemented in a FDIXP425 networkbased embedded system on a Linux platform. Experimental results prove the proposed scheme successfully performs across $Q R G$.
\end{abstract}

\section{Introduction}

Digital video technology has the potential to achieve much higher levels of quality and the technology is being improved at the increasing rate. Using compressed digital video however, less bandwidth was required and regular transmission became economical. Subsampling, which reduces the number of bits required to describe an image, is an efficient lossy compression technique $[2,3]$. Intra-frame compression is compression applied to still images and individual frames of a video sequence, and exploits the spatial redundancy within the image. By comparing adjacent pixels of the subsampled image at the receiving end, the value of the missing in-between pixels can be approximated. This process is known as interpolation. Interpolation can be used to make a subsampled image appear to have higher resolution than it actually has, and is usually more successful than pixel doubling. Subsampling can also be applied to video as an interframe compression technique, by transmitting only some of the frames. So the decoder would be required to interpolate the missing frames at the receiving end.

The residential gateway (RG) is the core device of the digital home network. Modern home network applications especially for real-time are demanding guaranteed Quality of Service (QoS) for bounded network bandwidth resources. In order to initiate a DiffServ-QoS (Differentiated Services-QoS) bandwidth management mechanism during network congestion, we proposed a QRG (QoS-aware Residential Gateway) with both real-time traffic monitoring, DiffServ-QoS and CBQ (Class Based Queuing) bandwidth management [1]. Applications with higher priority get the right to deliver first.

The rest of this paper is organized as follows. Section II covers the proposed adaptive scheme. The implementation and experimental results are presented in sections III. Finally conclusions and future work are summarized in section IV.

\section{The Proposed Adaptive Scheme}

For any incoming packet/traffic, QRG identify the traffic classes that are traveling through it. Here QRG classifies the traffic into but not limited to 7 classes as listed in Table 1, they are Security traffic, Multimedia traffic, ICMP traffic, File transfer traffic, Web traffic, Interactive traffic, and Best-effort traffic. Then DSME (DiffServ Marker Engine) features the matched traffic DiffSer-QoS enable [1]. Different traffic classes are 


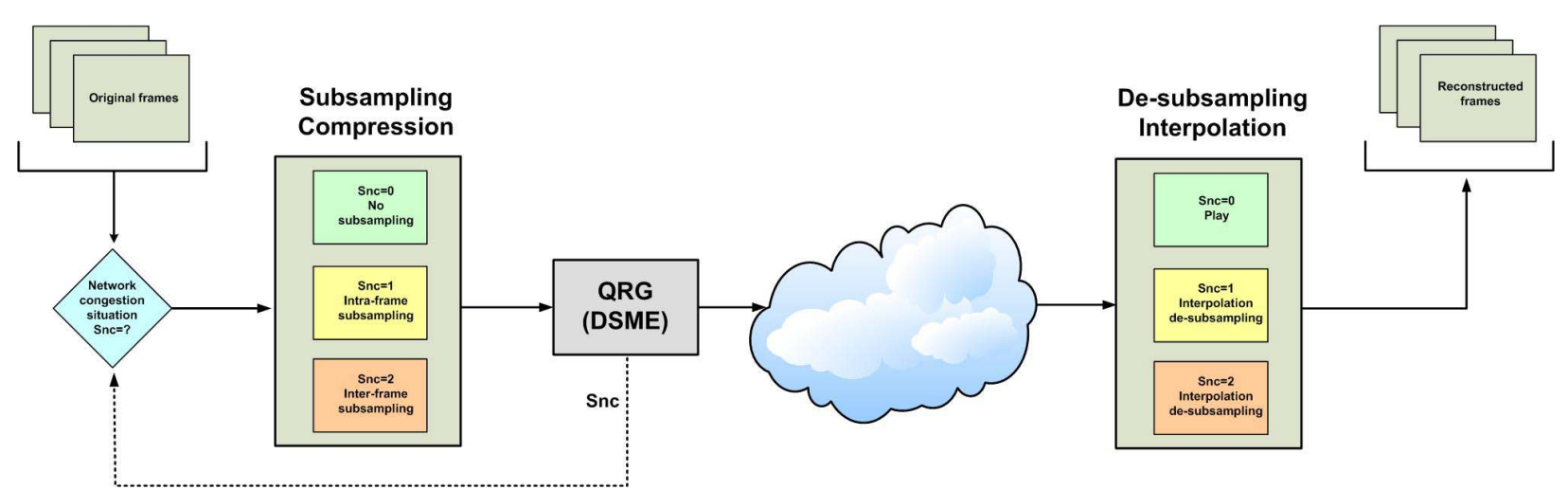

Figure 1. Block diagram of proposed adaptive compression scheme for video delivery over QRG

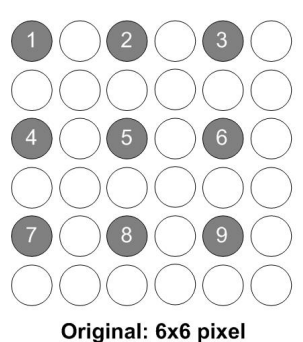

Figure 2. The adopted intra-frame subsampling/de-subsampling with interpolation algorithm

marked with different DiffServ CodePoint (DSCP) by DSME which is contained in the packet header. After that packets are forwarded according to the Per-Hop Behavior (PHB) treatment associated with the DiffServ CodePoint through the DiffServ-capable network.

In our scheme as shown in Fig. 1, if the immediate traffic is greater than the 5-min average, then it changes the congestion flag Snc from default zero to 1 . The congestion flag Snc will set to 2 if the immediate traffic is greater than the 5 -min average multiplied by $125 \%$. The congestion flag Snc feedback to the sender within the QRG-based home network or environment, so that the sender makes the right decision to select the suitable subsampling compression algorithm to meet the current network situation. At the receiving end, the receiving subsampled frames will be de-subsampled to reconstruct the image-frame according to the Snc.

If the congestion flag Snc set to 1, then intra-frame subsampling compression scheme is applied to individual frames of a video sequence. Intra-frame subsampling scheme could have variable compression ratio, e.g. selected pixels are selected as 4 to 1 or 2 to 1 subsampling patterns, what we adopted here is 4 to 1 subsampling pattern as shown in Fig. 2. The quality of the intra-frame subsampled image will be lower than the quality of the original. At the receiving end, the frames will be reconstructed with a simple linear interpolation as shown in Fig. 2, by comparing adjacent pixels of the subsampled image, the value of the missing in-between pixels can be approximated on a pixel-by-pixel basis due to the image locality property.

If the congestion flag Snc set to 2, then inter-frame subsampling compression scheme is used. The proposed inter-frame subsampling scheme not only discards data as intra-frame subsampling does, but also transmitting only contains the odd frames, i.e. frame 1 , 3,5 , etc. This leads to reduce the transmitting numbers of subsampled image to around a half of original. The effect of the whole video gets worse than intra-frame subsampling does. At the receiving end, the missing inbetween frames can be approximately reconstructed by comparing the previous and the next of the subsampled frames with a simple linear interpolation due to videotime locality property. After that the value of the missing in-between pixels of the frames can be reconstructed as the same way of intra-frame desubsampling interpolation does.

\section{Table 1. DiffServ-QoS setting for bandwidth} allocation for "user is out of home"

\begin{tabular}{|c|c|c|c|}
\hline Traffic class & $\begin{array}{c}\text { Protocol } \\
\text { categories }\end{array}$ & $\begin{array}{c}\text { Divert } \\
\text { port }\end{array}$ & $\begin{array}{c}\text { DiffSerV } \\
\text { setting }\end{array}$ \\
\hline Security traffic (HTTP:8088) & TCP:8088 & 9988 & EF \\
\hline $\begin{array}{c}\text { Web traffic } \\
\text { (HTTP:80) }\end{array}$ & TCP:80 & 9980 & AF23 \\
\hline File transfer traffic (FTP) & $\begin{array}{c}\text { TCP:21 } \\
\text { TCP:20 }\end{array}$ & $\begin{array}{c}9921 \\
9920\end{array}$ & $\begin{array}{c}\text { AF } 31 \\
\text { AF } 32\end{array}$ \\
\hline $\begin{array}{c}\text { Interactive traffic } \\
\text { (SSH, TELNET) }\end{array}$ & TCP:22 & 9922 & AF22 \\
TCP:25 & 9925 & AF22 \\
\hline Multimedia traffic (UDP) & UDP & 9990 & AF12 \\
\hline Ping (ICMP) & ICMP & 9910 & BE \\
\hline
\end{tabular}

\section{Implementation and Experiments}

We implemented the proposed QRG experimentally in an Intel $533 \mathrm{MHz}$ FDIXP425 network-based embedded system on a Linux platform. The testbed network layout and the physical installation are as 
shown in Fig. 3 and Fig. 4, respectively. The link between the two DiffServ routers, R1 and R2, is a 10 Mbps Ethernet link, i.e. a bottleneck, is composed of 6 isolated channels with the non-sharable bandwidths [1]. The marked EF and BE flows are assigned $2 \mathrm{Mbps}$, respectively. The marked $\mathrm{AF} 1 \mathrm{x}, \mathrm{AF} 2 \mathrm{x}, \mathrm{AF} 3 \mathrm{x}$, and AF4x flows are assigned 1.5 Mbps, respectively.

There are 2 experiments will be tested for the proposed scheme. QRG is always with DiffServ-QoS activation, so for example the UDP traffic is marked with AF12 flow for the "user is out" case during network congestion as shown in Table 1. In experiment 1, Sender2 and $\mathrm{H} 1$ respectively deliver UDP multimedia film by vlc (VideoLAN Client) to R2, which contains two different IPs for the two different senders. Later $\mathrm{H} 1$ sends background traffic (i.e. BE traffic) streams, $200 \mathrm{pkts} / \mathrm{s}$ (packets per second), 512 bytes/pkt (bytes per packet), to R2 to make network congestion occur. In experiment 2, H1 sends BE, AF31 and AF32 traffic streams, with the same $200 \mathrm{pkts} / \mathrm{s}$, 512 bytes/pkt respectively, to R2 to make normal (no drop) occur. Sender proceeds 50 consecutive frames from the Table Tennis sequence to simulate the proposed subsampling compression process, default $\mathrm{Snc}=0$ no subsampling for raw data in the case 1, $\mathrm{Snc}=1$ intra-frame subsampling in the case 2, and Snc $=2$ inter-frame subsampling in the case 3, respectively. The processed data sends to Receiver by FTP. Receiver de-subsamples the subsampled frame if detects, with a simple linear interpolation according to the Snc.

In experiment 1 , qdisc statistical analysis at $\mathrm{R} 1$ shows: Total traffic has 78102 packets (63897968 bytes), with 10254 packet drops, to be sent. BE Traffic is 63910 packets (44663396 bytes) with 10254 packet drops. AF12 traffic is 14163 packets (19233354 bytes) with 0 packet drops. This indicates the film displaying at $\mathrm{R} 2$ from $\mathrm{H} 1$ is affected strongly during network congestion, producing the low quality image in the lower one, while the film from Sender2 still keep the good quality of service in the upper one shown in Fig. 5.

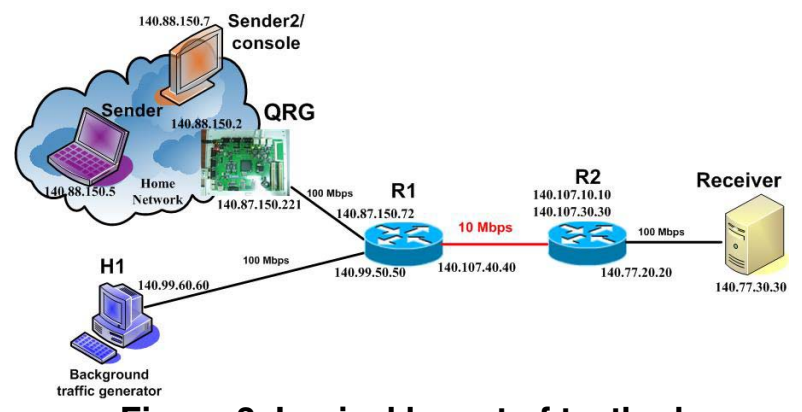

Figure 3. Logical layout of testbed

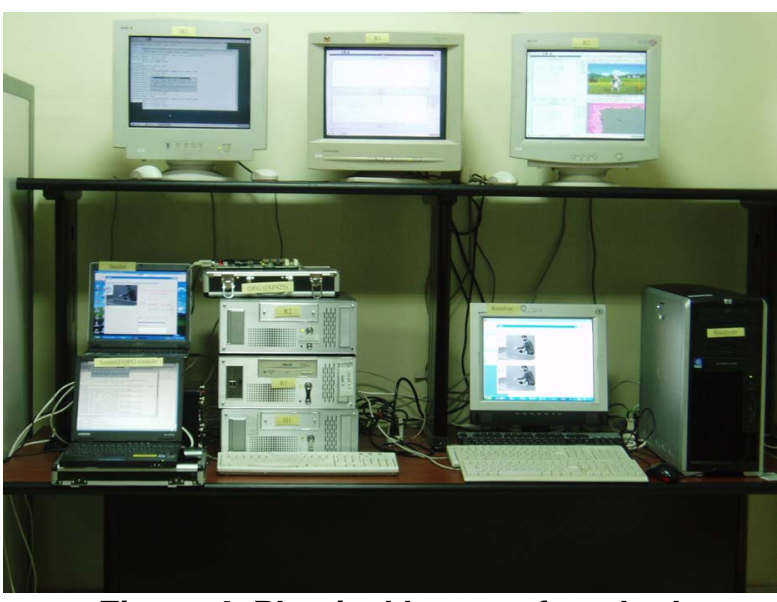

Figure 4. Physical layout of testbed

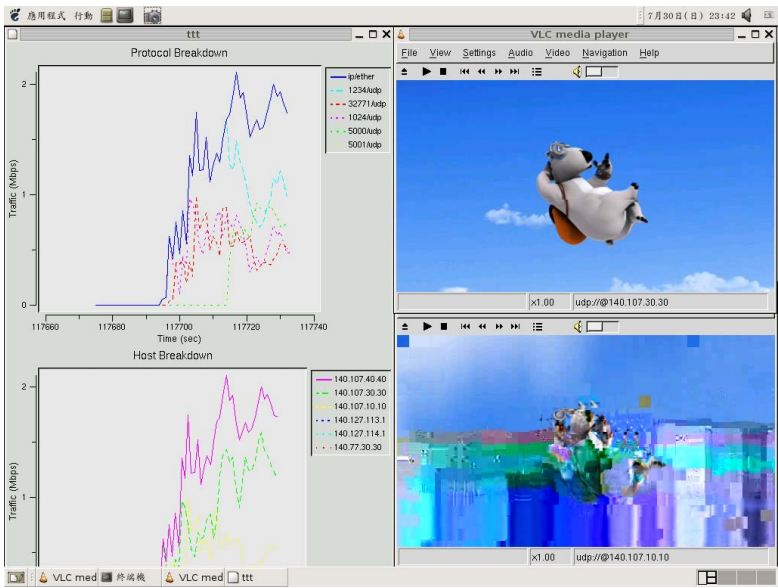

Figure 5. Film displayed at $\mathbf{R} 2$ in experiment 1: the upper/lower images with/without DiffServQoS activation during network congestion

There are 3 cases in experiment 2 . The result analysis is as listed in Table 2, and the detailed frame quality by frame-PSNR chart at Receiver is as shown in Fig. 6, Fig. 7, and Fig. 8, respectively. This indicates $\mathrm{Snc}=0$ got the best video quality, but it took more time (comparing 298 seconds in Snc=0, 148 seconds in $\mathrm{Snc}=1$, and 59 seconds in $\mathrm{Snc}=2$ ) for transmission, this means delay, drop rate became serious during network congestion. The receiver de-subsamples with a simple linear interpolation to reconstruct the subsampled image-frame in case 2 and case 3 . The upper image is the original frame, while the lower one is the reconstructed frame. The compression ratio is 3.890 and 7.781 in $\mathrm{Snc}=1$ and $\mathrm{Snc}=2$, respectively. The PeakSNR (PSNR) over all the frames in the whole video sequence is shown in Fig. 7 and Fig. 8, respectively. The quality of the subsampled image is lower than the quality of the original. It results in edges becoming blurred, but the acceptable video quality of consecutive 
frame is maintained. The results show that the proposed adaptive scheme worked well during network congestion.

\section{Table 2. Result analysis in experiment 2}

\begin{tabular}{|l|r|r|r|}
\hline \multicolumn{1}{|c|}{ Experiment 2 } & Case 1 (Snc=0) & Case 2 (Snc=1) & Case 3 (Snc=2) \\
\hline Total time (sec) & 298 & 148 & 59 \\
\hline $\begin{array}{l}\text { Total transmit } \\
\text { (frames/Byte) }\end{array}$ & $50 / 4264000$ & $50 / 1096000$ & $25 / 548000$ \\
\hline Compression Ratio & 1.000 & 3.890 & 7.781 \\
\hline PSNR (dB) & 999.999 (Fig. 6) & Fig. 7 & Fig. 8 \\
\hline $\begin{array}{l}\text { AF31 traffic } \\
\text { (packets/bytes) }\end{array}$ & $72351 / 39955175$ & $40564 / 22346211$ & $33282 / 18374400$ \\
\hline AF31 packet drops & 171 & 16 & 10 \\
\hline $\begin{array}{l}\text { AF32 traffic } \\
\text { (packets/bytes) }\end{array}$ & $63723 / 38645710$ & $41189 / 23442482$ & $33597 / 18930638$ \\
\hline AF32 packet drops & 4651 & 642 & 333 \\
\hline
\end{tabular}

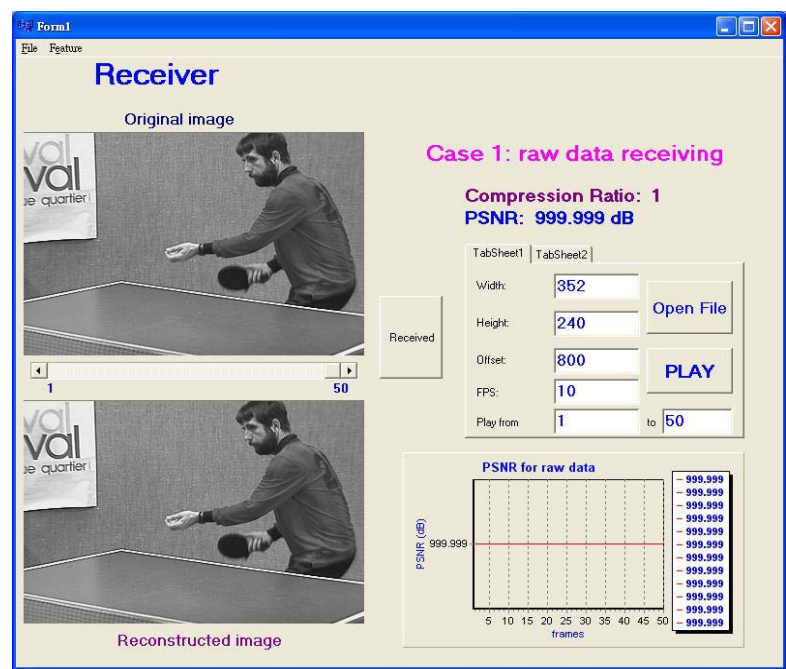

Figure 6. Snc $=0$ in the case 1 of experiment 2

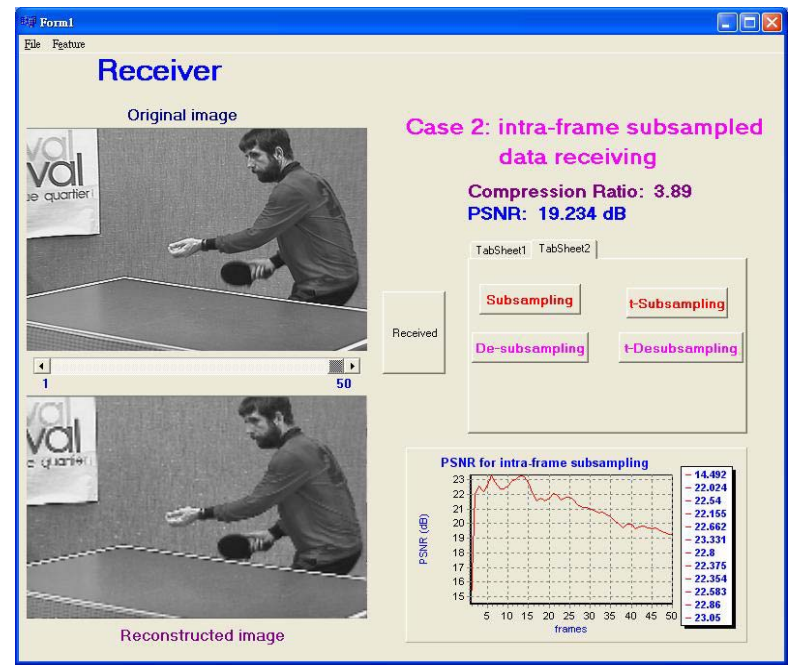

Figure 7. Snc=1 in the case 2 of experiment 2

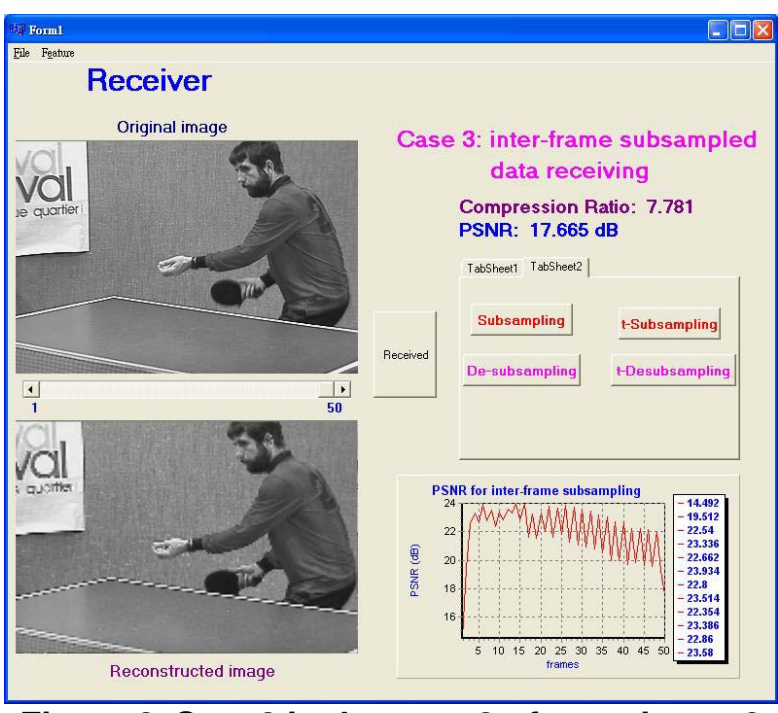

Figure 8. Snc=2 in the case 3 of experiment 2

\section{Conclusions and Future Work}

Subsampling is the most basic of lossy compression technique. The proposed subsampling scheme can be adaptively adjust with variable compression ratio according to the feedback information from the FDIXP425-QRG to meet the network demand during network congestion. Experimental results show that the proposed scheme successfully performs across QRG, so that good quality of service and video performance are maintained.

Our future work will add network security features to QRG, and develop the novel compression scheme for video delivery smoothly and quickly across the network to the receiver.

\section{References}

[1] Wen-Shyang Hwang and Pei-Chen Tseng, "A QoS-aware Residential Gateway with Bandwidth Management," IEEE Transactions on Consumer Electronics, Vol. 51, No. 3, pp.840-848, August 2005.

[2] Yu-Wen Huang, Bing-Yu Hsieh, Tung-Chien Chen, and Liang-Gee Chen, "Analysis fast algorithm and VLSI architecture design for H.264 AVC intra frame coder," IEEE Transactions on Circuits and Systems for Video Technology, Vol. 15, No. 3, pp.378-401, March 2005.

[3] Christopher E. Cramer and Erol Gelenbe, "Video quality and traffic QoS in learning-based subsampled and receiverinterpolated video sequences," IEEE Journal on selected areas in communications, Vol. 18, No. 2, pp.150-167, February 2000. 